Homology, Homotopy and Applications, vol.22(2), 2020, pp.295-321

\title{
BRAIDED CATEGORICAL GROUPS AND STRICTIFYING ASSOCIATORS
}

\author{
OLIVER BRAUNLING
}

\author{
(communicated by Emily Riehl)
}

\begin{abstract}
A key invariant of a braided categorical group is its quadratic form, introduced by Joyal and Street. We show that the categorical group is braided equivalent to a simultaneously skeletal and strictly associative one if and only if the quadratic form comes from a bilinear form. This generalizes the result of JohnsonOsorno that all Picard groupoids can simultaneously be strictified and skeletalized, except that in the braided case there is a genuine obstruction.
\end{abstract}

\section{Introduction}

A braided monoidal category is always braided monoidal equivalent to a skeletal one, and always braided monoidal equivalent to a strictly associative one. However, typically it is impossible to achieve both properties simultaneously; even in the more restrictive symmetric monoidal case. But Johnson and Osorno [JO12] have shown that this problem, surprisingly, disappears for Picard groupoids, i.e. symmetric categorical groups. We generalize this to the braided case, except that here one faces an obstruction. In detail:

A braided categorical group is a braided monoidal category $(C, \otimes)$ which is additionally a groupoid and for all objects $X \in \mathrm{C}$, there exists an object $X^{-1}$ with an arrow

$$
X \otimes X^{-1} \stackrel{\sim}{\longrightarrow} \mathbf{1}
$$

to the unit object. The same concept is also called a 'braided $G r$-category' or 'braided weak 2-group'. If the braiding is symmetric, it is called a Picard groupoid.

Joyal and Street [JS93] give a classification of braided categorical groups up to braided monoidal equivalences. They attach to any such an abelian 3-cocycle in $H_{a b}^{3}\left(\pi_{0} \mathrm{C}, \pi_{1} \mathrm{C}\right)$. Eilenberg-Mac Lane canonically identify the latter group with the group of quadratic forms

$$
\operatorname{tr}: H_{a b}^{3}(G, M) \stackrel{\cong}{\longrightarrow} \operatorname{Quad}(G, M)
$$

This isomorphism holds for arbitrary abelian groups $G, M$. The subtle differences

The author was supported by DFG GK1821 "Cohomological Methods in Geometry".

Received November 27, 2019, revised January 30, 2020; published on May 13, 2020.

2010 Mathematics Subject Classification: 18D10, 19 D23.

Key words and phrases: braided categorical group, Picard groupoid, strictification, skeletalization, associator.

Article available at http://dx.doi.org/10.4310/HHA.2020.v22.n2.a19

Copyright (C) 2020, International Press. Permission to copy for private use granted. 
between symmetric bilinear forms and quadratic forms cause some intricacies here unless 2 is invertible, and this issue lies at the heart of the entire matter. Our main result is as follows.

Theorem A. A braided categorical group $(\mathrm{C}, \otimes)$ is braided monoidal equivalent to one which is simultaneously strictly associative and skeletal if and only if its quadratic form comes from a bilinear form, i.e. $q(x)=S(x, x)$, for some not necessarily symmetric bilinear form $S$.

See Theorem 4.15. This generalizes the following result and reproves it in a different way.

Theorem B (Johnson-Osorno [JO12]). Every Picard groupoid (C, $\otimes)$ is symmetric monoidal equivalent to one which is simultaneously strictly associative and skeletal.

We call a quadratic form polar if its polarization, i.e. the symmetric bilinear form

$$
b(x, y):=q(x+y)-q(x)-q(y),
$$

is of the shape $t(x, y)+t(y, x)$ for some bilinear form $t: G \times G \rightarrow M$ (where $t$ is not required to be symmetric or non-degenerate in any way). This property turns out to be equivalent to the existence of a (usually different) bilinear form $S$ such that $q(x)=S(x, x)$ (Corollary 4.14). Theorem B now follows from our main result since for Picard groupoids the polarization always vanishes, so one can just take $t(x, y):=0$. We give explicit examples of non-polar forms. Even in the non-polar situation, one can always add new objects to the category to make it polar.

Theorem C. For every braided categorical group $(\mathrm{C}, \otimes)$, there exists an essentially surjective and faithful (but typically not full) braided monoidal functor from another braided categorical group

$$
(\widehat{\mathrm{C}}, \otimes) \longrightarrow(\mathrm{C}, \otimes),
$$

surjective on $\pi_{0}$, and an isomorphism on $\pi_{1}$, such that $(\widehat{\mathrm{C}}, \otimes)$ is simultaneously strictly associative and skeletal. We call $(\widehat{\mathrm{C}}, \otimes)$ a polar cover of $(\mathrm{C}, \otimes)$.

See Theorem 4.16. This construction is not canonical. It has occasionally been asked $^{1}$ how to find the commutativity constraint explicitly if one is only given the quadratic form. We answer this in the polar case.

Theorem D. Let $G, M$ be abelian groups. Let $\left(\beta_{i}\right)_{i \in I}$ be a basis of the $\mathbb{F}_{2}$-vector space $G / 2 G$. Let $q \in \operatorname{Quad}(G, M)$ be a polar quadratic form and pick a $t: G \times G \rightarrow M$ such that $b(x, y)=t(x, y)+t(y, x)$. Then

$$
\bar{q}(x):=q(x)-t(x, x)
$$

defines an element $\bar{q} \in \operatorname{Hom}_{\mathbb{Z}}(G / 2 G, M)$ and each $\bar{q}\left(\beta_{i}\right)$ is 2-torsion in $M$. Define the

\footnotetext{
1 "But I had difficulty seeing in an explicit way how to get an associator and braiding from a quadratic form." [Var]. Quinn [Qui99, §2.5] or Galindo-Jaramillo [GJ16, §4.4] discuss $G$ finite and $M=U(1)$. Various further results exist in the literature, mostly for $G$ finite and $M$ inside $\mathbb{C}^{\times}$, Wall [Wal63], Durfee [Dur77].
} 
abelian 3-cocycle $(h, c) \in H_{a b}^{3}(G, M)$ with

$$
\begin{aligned}
h(x, y, z) & :=0 \\
c(x, y) & :=t(x, y)+\sum_{i \in I} \bar{x}_{i} \cdot \bar{y}_{i} \cdot \bar{q}\left(\beta_{i}\right),
\end{aligned}
$$

where $\bar{x}, \bar{y}$ are the vectors we get under the quotient map $G \rightarrow G / 2 G$, spelled out with respect to the basis $\left(\beta_{i}\right)$. This means that $\bar{x}_{i}, \bar{y}_{i} \in \mathbb{F}_{2}$. Then $(h, c)$ maps to $q$ under the isomorphism of Equation 1.1.

Note that while [JO12] abstractly proves that Picard groupoids can be made strictly associative and skeletal by showing that the symmetric 3-cohomology class must have a representative with trivial associator, the above provides an explicit 3 -cocycle having this property.

When preparing this text, I sometimes got stuck because in a lot of literature many little (and often admittedly harmless) verifications are left as Exercises, especially in the older literature, where perhaps most of this was considered folklore. I found this a little inconvenient, so this text is written in a quite self-contained way, providing details for many of these customary omissions.

\section{Acknowledgments}

I thank Niles Johnson and Brad Drew for helpful correspondence. I am also thankful for a number of clarifications in the proofs which were suggested by the referee.

\section{Braided categorical groups}

Definition 2.1. A braided categorical group is a braided monoidal category $(C, \otimes)$ which is additionally a groupoid and for every object $X \in \mathrm{C}$, we are given an object $X^{-1}$ with an arrow

$$
\varepsilon_{X}: X \otimes X^{-1} \stackrel{\sim}{\longrightarrow} \mathbf{1}
$$

to the unit object. Alternative names are: braided $G r$-categories, braided weak 2groups.

It may appear more natural to demand the existence of an inversion functor

$$
(-)^{-1}: \mathrm{C}^{o p} \longrightarrow \mathrm{C}
$$

sending $X$ to $X^{-1}$ functorially. The definition above does not a priori guarantee any functoriality in the formation of inverses. However, Laplaza has shown that in any categorical group the inverses $X^{-1}$ necessarily extend uniquely to give a functor as in Equation 2.2, [Lap83, Proposition 4.3]. This is also discussed in [BL04].

Definition 2.2. If the braiding

$$
s_{X, Y}: X \otimes Y \stackrel{\sim}{\longrightarrow} Y \otimes X
$$

is symmetric, i.e. $s_{Y, X} \circ s_{X, Y}=\mathrm{id}_{X \otimes Y}$ for all $X, Y \in \mathrm{C}$, then (C, $\left.\otimes\right)$ is called a Picard groupoid. 
The definition of braided categorical groups goes back to Joyal and Street [JS93, $\S 3]$.

Given braided categorical groups $P, P^{\prime}$, we write $\operatorname{Hom}^{\otimes}\left(P, P^{\prime}\right)$ for the category of braided monoidal functors from $\mathrm{P}$ to $\mathrm{P}^{\prime}$ : Objects are functors $\mathrm{P} \rightarrow \mathrm{P}^{\prime}$ preserving the monoidal structure along with the braiding and associativity constraints; morphisms (usually called "homotopies") are natural transformations between such functors, which, however, also need to be compatible with the braided monoidal structure. A fully spelled out definition of braided monoidal functors is given for example in [CK07, §6, take $G=1$ ].

Remark 2.3 (Notation). Some variation in language exists. In Deligne's work [Del87, $\S 4.1]$ Picard groupoids are called 'commutative Picard categories', while in [Bre11, $\S 2.1]$ merely 'Picard categories'. Monoidal categories are often called tensor categories instead.

\subsection{General skeletalization and strictification methods}

A braided categorical group $(\mathrm{C}, \otimes)$ has three key invariants:

1. $\pi_{0}(\mathrm{C}, \otimes)$, the set of isomorphism classes of objects; an abelian group,

2. $\pi_{1}(\mathrm{C}, \otimes)$, the automorphism group of the unit object $\mathbf{1}_{\mathrm{C}}$; another abelian group,

3. the map $q: \pi_{0}(\mathrm{C}, \otimes) \rightarrow \pi_{1}(\mathrm{C}, \otimes)$ which assigns to any object $X \in \mathrm{C}$ its signature

$$
q([X]):=s_{X, X} \otimes X^{-1} \otimes X^{-1},
$$

which is an automorphism of the unit $\mathbf{1}_{\mathrm{C}}$. Here $s_{X, X}$ denotes the braiding

$$
s_{X, X}: X \otimes X \stackrel{\sim}{\longrightarrow} X \otimes X .
$$

This is an automorphism of $X \otimes X$, and along the contractions $\varepsilon_{X}$ of Equation 2.1 induces one of $\mathbf{1}_{\mathrm{C}}$, thus giving an element of $\pi_{1}(\mathrm{C}, \otimes)$.

There are particularly nice types of braided categorical groups:

Definition 2.4. A category $\mathrm{C}$ is called skeletal if for any isomorphic objects $X, X^{\prime} \in \mathrm{C}$ we must have $X=X^{\prime}$, i.e.

$$
X \simeq X^{\prime} \quad \Rightarrow \quad X=X^{\prime} .
$$

Definition 2.5. A braided categorical group $(C, \otimes)$ is called strict (but we usually write strictly associative ${ }^{2}$ ) if the associativity constraint

$$
a_{X, Y, Z}: X \otimes(Y \otimes Z) \stackrel{\sim}{\longrightarrow}(X \otimes Y) \otimes Z
$$

and the unit constraints

$$
\mathbf{1}_{\mathrm{C}} \otimes X \stackrel{\sim}{\longrightarrow} X \quad \text { and } \quad X \otimes \mathbf{1}_{\mathrm{C}} \stackrel{\sim}{\longrightarrow} X
$$

are all identity maps (and, in particular, the objects on the left and right of these isomorphisms are the same). If the braiding is symmetric, one also calls such a $(\mathrm{C}, \otimes)$ a permutative category.

\footnotetext{
${ }^{2}$ Sometimes people use the word "strict" also to imply that the commutativity constraint would have to be trivial, which would be much more restrictive.
} 
Both of these properties do not really occur in nature much.

Example 2.6. Let $k$ be a field. The category $\left(\operatorname{Vect}_{f}(k), \otimes\right)$ of $k$-vector spaces with the usual tensor product has neither property. In particular, if one honestly follows the definitions,

$$
k^{\ell} \otimes\left(k^{n} \otimes k^{m}\right) \stackrel{\sim}{\longrightarrow}\left(k^{\ell} \otimes k^{n}\right) \otimes k^{m}
$$

is an isomorphism between two genuinely different objects.

This example is too basic, and in many ways not really helpful. The associativity constraint does such a truly basic and light thing in this example that it is really hard to imagine that anything could ever go wrong or that it is truly worth keeping the associativity constraint in mind.

As this is a critical possible misconception, we shall dwell on this for a bit, even though every category theory inclined reader may shake their head in boredom. We shall look at some non-trivial examples soon.

But first, we point out two basic simplication procedures for arbitrary braided categorical groups:

Theorem 2.7. Every braided monoidal category is braided monoidal equivalent to a skeletal one. We call this skeletalization.

Theorem 2.8 (Mac Lane-Isbell). Any given braided monoidal category is braided monoidal equivalent to a strictly associative one. We call this strictification.

We shall sketch both proofs below, in order to see what can go wrong. However, aside from proving these, and this is crucial, in general it is absolutely impossible to achieve being strictly associative and skeletal simultaneously.

Proof sketch for Theorem 2.7. Any category is equivalent to a skeletal one: Given C, (as Step 1) pick precisely one object $X$ from any isomorphism class. Further, for any object in C, pick (as Step 2) a fixed isomorphism to the single chosen object representing its isomorphism class. Now consider the full subcategory $C^{\prime}$ of these objects. It is, by construction, skeletal. The inclusion $\mathrm{C}^{\prime} \rightarrow \mathrm{C}$ is clearly a fully faithful functor, but it is also essentially surjective by construction. Now we make $C^{\prime}$ braided monoidal: For any $X, Y \in \mathrm{C}^{\prime}$ we have $X \otimes_{\mathrm{C}} Y$ in $\mathrm{C}$, and there will be precisely one object $C_{X, Y} \in \mathrm{C}^{\prime}$ in the same isomorphism class (by Step 1). Define $X \otimes_{\mathrm{C}^{\prime}} Y:=C_{X, Y}$. Define the associativity and commutativity constraint by pre- and postcomposing the ones in $\mathrm{C}$ with the fixed isomorphisms (of Step 2) to the fixed objects representing the isomorphism classes of $C$ uniquely in $C^{\prime}$.

We gave the proof because it makes clear how this procedure can mess up the associativity and commutativity constraints, even if they were easy maps in the original category.

Example 2.9. In the notation of the proof, given $X, Y \in \mathrm{C}$, we obtain objects $C_{X, Y}$ and $C_{Y, X}$ in $C^{\prime}$ and the braiding becomes

$$
C_{X, Y} \stackrel{\sim}{\longrightarrow} X \otimes Y \underset{s_{X, Y}}{\stackrel{\sim}{\longrightarrow}} Y \otimes X \stackrel{\sim}{\longleftarrow} C_{Y, X},
$$

where $s_{X, Y}$ is the original braiding of C. Since our category $\mathrm{C}^{\prime}$ is skeletal, we must have $C_{X, Y}=C_{Y, X}$, so the composite map is just an automorphism of $C_{X, Y}$. However, 
since we have no overall control of the two outer isomorphisms in Equation 2.3 (the ones chosen in Step 2 of the proof sketch above), it is by no means clear that this is the identity automorphism.

But one may believe that a more careful construction in the proof would be able to solve this problem. This is not so. Although well-documented in the literature, let us have a look at Isbell's highly instructive counterexample, which immediately crushes all hope.

Theorem 2.10 (Isbell [Isb69]). Let $\mathrm{C}$ be a category which has all products. Suppose there is some object $X$ such that $X \times X \simeq X$ and $\operatorname{End}(X)$ has at least two elements. Make $\mathrm{C}$ a symmetric monoidal category using the product, i.e. $X \otimes Y$ is a product of $X, Y$. Then $(\mathrm{C}, \otimes)$ is not monoidal equivalent to any simultaneously strictly associative and skeletal monoidal category.

Example 2.11. The category of sets or the category of all $k$-vector spaces are examples. The category of all finite-dimensional $k$-vector spaces is not an example (see Example 2.12).

Proof. For a product of objects $X, Y$, we write $p_{X}^{X, Y}$ resp. $p_{Y}^{X, Y}$ for the two projections. Let $f, g, h: X \rightarrow X$ be arbitrary morphisms. We consider the following four composite morphisms:

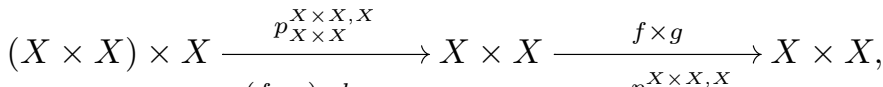

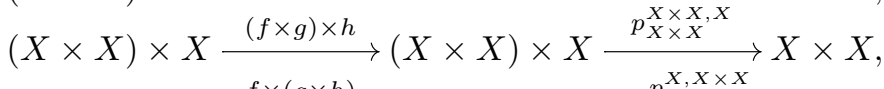

$$
\begin{aligned}
& X \times(X \times X) \stackrel{f \times(g \times h)}{\underset{p_{X}^{X}, X \times X}{\longrightarrow}} X \times(X \times X) \stackrel{p_{X}^{X, X \times X}}{\longrightarrow} X,
\end{aligned}
$$

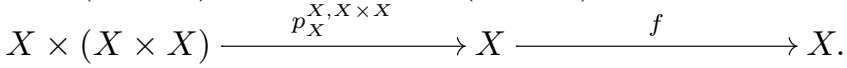

Note that the first and the second composite morphism agree on the nose. Moreover, the third and the fourth composite morphism agree. Proof by contradiction: Assume $C$ is monoidal equivalent to a strictly associative skeletal category $C^{\prime}$, say by some functor $F$. We apply this functor and consider the four morphisms above. For simplicity, let us from now on write $X$ instead of $F(X)$; or equivalently without loss of generality assume $C$ had been $C^{\prime}$ to start with. Two new things happen: Since our target category is strictly associative, the associativity constraint $X \times(X \times X) \longrightarrow(X \times X) \times X$ is between the same objects and the identity map. Since the monoidal bifunctor is natural in each variable, it follows that the second and third composite morphism simplify to

$$
\begin{aligned}
& X \times X \times X \stackrel{f \times g \times h}{\longrightarrow} X \times X \times X \underset{f \times g \times h}{\longrightarrow} X \times X \times X \stackrel{p_{X \times X}^{X \times X, X}}{\longrightarrow} X \times X, \\
& X \times X \times X \stackrel{p_{X}^{X, X \times X}}{\longrightarrow} X
\end{aligned}
$$

and the left arrows in both lines agree. However, since the category is skeletal, $X \times X \cong X$ means that these are the same object. This means that all first factor projections of the products here agree with $p_{X}^{X, X}$, using that our equivalence of categories has preserved products. Thus, also the second arrows in both lines above 
agree. But this means that the composites agree. Hence, all four morphisms in Equation 2.4 are the same. Moreover, $X \times X \times X=X$. Thus, comparing the first with the last composite in Equation 2.4, we obtain

$$
f \circ p_{X}^{X, X}=(f \times g) \circ p_{X}^{X, X}
$$

and this is valid for arbitrary choices of $f, g, h$ above. As $p_{X}^{X, X}$ is an epic, we deduce $f=f \times g$. Swapping the rôles of $f, g$, we also obtain $f \times g=g$, and then $f=g$ for arbitrary $f, g$, contradicting that $f, g \in \operatorname{End}(X)$ can be chosen different by assumption.

Example 2.12. The category of all finite-dimensional $k$-vector spaces with the direct $\operatorname{sum}\left(\operatorname{Vect}_{f}(k), \oplus\right)$ is symmetric monoidal equivalent to a both strictly associative and skeletal symmetric monoidal category. As objects for our strictly associative skeletal $\mathrm{C}^{\prime}$ take $\mathbb{Z}_{\geqslant 0}$ and define $n \boxplus m:=n+m, \operatorname{Hom}_{\mathrm{C}^{\prime}}(n, m):=\operatorname{Hom}_{k}\left(k^{n}, k^{m}\right)$ with the identity commutativity constraint. Then $F:\left(C^{\prime}, \boxplus\right) \rightarrow\left(\operatorname{Vect}_{f}(k), \oplus\right)$ sending $n$ to $k^{n}$ is a symmetric monoidal equivalence. One can try to extend this to allowing countably infinite dimension. As objects take $\mathbb{Z}_{\geqslant 0} \cup\{\infty\}$ and define $n \boxplus \infty:=\infty$ and $\infty \boxplus \infty:=\infty$, and take $F(\infty):=k^{\oplus \infty}$. Given $f, g \in \operatorname{Hom}_{k}\left(k^{\infty}, k^{\infty}\right)$, it is unclear how to define

$$
f \oplus g \in \operatorname{Hom}_{k}\left(k^{\infty}, k^{\infty}\right),
$$

leading us back to Isbell's counterexample.

Now, let us quickly look at how to make a braided monoidal category strictly associative.

Proof sketch for Theorem 2.8. Let $X$ be the free monoid generated by the objects of C, i.e. objects are finite formal words

$$
\text { " } x_{1} x_{2} \ldots x_{m} ",
$$

where the letters " $x_{1}, \ldots, x_{m}$ " are objects $x_{i} \in \mathrm{C}$. The empty word is also allowed. We define a category $\mathrm{C}^{\prime}$ by having $X$ as its objects, and define on objects

$$
\begin{aligned}
\psi: \mathrm{C}^{\prime} & \longrightarrow \mathrm{C}, \\
" x_{1} x_{2} \ldots x_{m} " & \left.\left.\longmapsto\left(\cdots\left(x_{1} \otimes x_{2}\right) \otimes \cdots\right) \otimes x_{m-1}\right) \otimes x_{m}\right),
\end{aligned}
$$

i.e. we apply the monoidal bifunctor to " $x_{1} x_{2} \ldots x_{m}$ ", bracketed all to the left. The empty word goes to $\mathbf{1}_{\mathrm{C}}$, a single letter " $x_{1}$ " to the object it represents, " $x_{1} x_{2}$ " to $x_{1} \otimes x_{2}$, " $x_{1} x_{2} x_{3}$ " to $\left(\left(x_{1} \otimes x_{2}\right) \otimes x_{3}\right)$ and so on. We equip $C^{\prime}$ with the structure of a category by demanding that the above should be a fully faithful functor. This means that

$$
\operatorname{Hom}_{\mathrm{C}^{\prime}}\left(\text { " } x_{1} x_{2} \ldots x_{m} \text { ", " } y_{1} y_{2} \ldots y_{n} "\right):=\operatorname{Hom}_{\mathrm{C}}\left(\psi\left(x_{1} x_{2} \ldots x_{m}\right), \psi\left(y_{1} y_{2} \ldots y_{n}\right)\right)
$$

Define a functor $\psi^{\prime}: \mathrm{C} \rightarrow \mathrm{C}^{\prime}$ in the opposite direction by sending the object $x$ to the single letter word " $x$ ". This gives an equivalence of categories. Define a braided monoidal structure on $C^{\prime}$ by simply concatenating words,

$$
\text { " } x_{1} x_{2} \ldots x_{m} " \otimes " y_{1} y_{2} \ldots y_{n} ":=" x_{1} x_{2} \ldots x_{m} y_{1} y_{2} \ldots y_{n} ",
$$

and compatibly on morphisms (as the Hom-sets agree with the input category C, one can import this part of the structure, see Equation 2.5). Let the empty word " "act 
as the tensor unit $\mathbf{1}_{\mathrm{C}^{\prime}}$. Since we really just concatenate words, it is clear that

$$
\mathbf{1}_{\mathrm{C}^{\prime}} \otimes " x_{1} x_{2} \ldots x_{m} "=" x_{1} x_{2} \ldots x_{m} ",
$$

and correspondingly for " $x_{1} x_{2} \ldots x_{m}$ " $\otimes \mathbf{1}_{\mathrm{C}^{\prime}}$, so $\left(\mathrm{C}^{\prime}, \otimes\right)$ indeed is a strictly associative braided monoidal category. Finally, one has to check that the equivalence of categories $\psi$ is braided monoidal. See [JS93] for a complete argument.

We see that the above procedure increases the cardinality of pairwise isomorphic objects massively.

Example 2.13. If we strictify associativity in $\left(\operatorname{Vect}_{f}(k), \oplus\right)$ using the procedure of the above proof, objects will be finite words of vector spaces " $x_{1} x_{2} \ldots x_{m}$ " which end up being isomorphic if and only if $\sum \operatorname{dim} x_{i}=\sum \operatorname{dim} x_{i}^{\prime}$. Thus, we get a very different category from the strictly associative one in Example 2.12.

We also see that alternating between this strictification and skeletalization procedure will just yield more and more complicated categories, which rather carry us farther away from our goal of having a simultaneously skeletal and strictly associative model.

\section{Strictification}

\subsection{Abelian and symmetric cohomology}

In order to dig a little deeper into these problems, we should first recall two "cohomology theories' (of sorts). We first need to recall how group cohomology can be defined, from two different viewpoints. Let $G$ be a group and $M$ a $G$-module. In pure algebra, group cohomology is usually defined as

$$
H_{g r p}^{n}(G, M):=\operatorname{Ext}_{\mathbb{Z}[G]}^{n}(\mathbb{Z}, M)
$$

in the category of $\mathbb{Z}[G]$-modules, where $\mathbb{Z}[G]$ is the group ring. Since $\operatorname{Ext}_{\mathbb{Z}[G]}^{0}(\mathbb{Z}, M)=$ $\operatorname{Hom}_{\mathbb{Z}[G]}(\mathbb{Z}, M)=M^{G}$, these Ext-groups then correspond to the right-derived functor groups $\mathbf{R}^{n}(-)^{G}$ of the left-exact functor of taking $G$-invariants. In topology one may prefer a different take on the same concept: Write $K(M, n)$ for the $n$-th EilenbergMac Lane space with single non-zero homotopy group $M$ in degree $n$. If $M$ has the trivial $G$-module structure, one can also define group cohomology as

$$
H_{g r p}^{n}(G, M):=[K(G, 1), K(M, n)],
$$

the pointed homotopy classes of maps from $K(G, 1)$ (which is nothing but the classifying space of the group).

The topological as well as the algebraic approach to the definition are equivalent and give the same cohomology groups. While we mostly work with an algebraic viewpoint below, the topologist's definition makes it much easier to motivate the construction of two other cohomology theories:

Definition 3.1 (Eilenberg-Mac Lane). Let $G$ be an abelian group and $M$ an abelian group (regarded as a trivial $G$-module). 
1. The groups

$$
H_{a b}^{n}(G, M):=[K(G, 2), K(M, n+1)]
$$

are called abelian cohomology.

2. The groups

$$
H_{\text {sym }}^{n}(G, M):=[K(G, 3), K(M, n+2)]
$$

are called symmetric cohomology.

We can quickly motivate where these definitions originate from: There are equivalences of homotopy categories, due to Joyal-Tierney (following ideas of Grothendieck),

$$
\begin{aligned}
& H o \text { (categorical groups) }\longrightarrow H o \text { (homotopy }[1,2] \text {-types }), \\
&H o \text { (braided categorical groups })\longrightarrow H o \text { (homotopy }[2,3] \text {-types }), \\
&H o \text { (Picard groupoids }) \longrightarrow H o(\text { homotopy }[n, n+1] \text {-types) } \\
&\text { (for any } n \geqslant 3),
\end{aligned}
$$

where on the right we refer to pointed unstable homotopy types. In each case the objects on the right-hand side are determined by providing the groups $G, M$ forming the two consecutive non-trivial $\pi_{n}, \pi_{n+1}$ as well as a cocycle corresponding to the corresponding Postnikov invariant, and concretely the relevant $k$-invariant lies in

$$
H^{3}(G, M), \quad \text { resp. } \quad H_{a b}^{3}(G, M), \quad \text { resp. } \quad H_{\text {sym }}^{3}(G, M),
$$

depending on which of the three cases one considers. In Equation 3.1 the functor inducing the equivalence is always a suitable kind of nerve. We refer to [BCC93], [CC96], [GMdR02], [JO12] for details on this story. We shall see this in a little more detail in $\S 3.2$ in the braided case, which is the only instance of the above relevant for our purposes.

While introducing the cohomology groups of Definition 3.1 in the above topological language is nice conceptually, it does not help much for computations. For group cohomology one can use the standard Chevalley-Eilenberg projective resolution of the trivial $G$-module $\mathbb{Z}$ as a $\mathbb{Z}[G]$-module in order to compute $\operatorname{Ext}_{\mathbb{Z}[G]}^{n}(\mathbb{Z}, M)$. When using the quasi-isomorphic complex of normalized cocycles $P \bullet$ (so that the complex

$$
\left(\operatorname{Hom}_{\mathbb{Z}[G]}(P \bullet, M), \partial^{\bullet}\right)
$$

is the one which is frequently called the complex of 'inhomogeneous chains', e.g., [NSW08, Chapter I, §2]) instead, a group 3-cocycle is a map

$$
h: G^{3} \longrightarrow M
$$

such that $\partial h=0$, which unravels as the condition

$$
h(x, y, z)+h(u, x+y, z)+h(u, x, y)=h(u, x, y+z)+h(u+x, y, z) .
$$

A group 3-coboundary is $\partial k$ for some $k: G^{2} \rightarrow M$ such that $h$ has the shape

$$
h(x, y, z)=k(y, z)-k(x+y, z)+k(x, y+z)-k(x, y) .
$$

These explicit expressions can directly be unravelled from [NSW08, Chapter I, §2] for example.

In the literature on symmetric or braided monoidal categories, one often uses the following additional condition: 
Definition 3.2. An inhomogeneous chain $h: G^{n} \rightarrow M$ is called normalized if we have $h\left(x_{1}, \ldots, x_{n}\right)=0$ as soon as $x_{i}=0$ for some $i$. fact.

One can always restrict to considering normalized cochains in view of the following

Lemma 3.3. Given an inhomogeneous chain $h: G^{n} \rightarrow M$, there exists a normalized inhomogeneous chain $h^{\prime}: G^{n} \rightarrow M$ such that $[h] \equiv\left[h^{\prime}\right] \in H^{n}(G, M)$ both represent the same group cohomology class.

Proof. This corresponds to using the normalized versus the unnormalized bar complex, see [Wei94, Chapter 6, §6.5.5] for background. For a direct proof without going back to the formalism of bar complexes, one can follow [NSW08, Chapter I, §2, Exercise 5].

Next, following Eilenberg and Mac Lane we discuss the analogous explicit expressions for abelian and symmetric cohomology.

Definition 3.4. Let $G, M$ be abelian groups.

1. An abelian 3-cocycle is a pair $(h, c)$ consisting of a group 3-cocycle $h: G^{3} \rightarrow M$ such that

$$
h(x, 0, z)=0
$$

and a map $c: G^{2} \rightarrow M$ which satisfies

$$
\begin{aligned}
h(y, z, x)+c(x, y+z)+h(x, y, z) & =c(x, z)+h(y, x, z)+c(x, y), \\
-h(z, x, y)+c(x+y, z)-h(x, y, z) & =c(x, z)-h(x, z, y)+c(y, z),
\end{aligned}
$$

for all $x, y, z \in G$.

2. An abelian 3-coboundary is a group 3-cocycle $h=\partial k$ for some map $k: G^{2} \rightarrow M$ with $k(x, 0)=0$ and $k(0, y)=0$. It can be interpreted as an abelian 3 -cocycle $(h, c)$ using

$$
c(x, y):=k(x, y)-k(y, x) .
$$

Note that every abelian 3-coboundary is indeed an abelian 3-cocycle, e.g.

$$
h(x, 0, z)=k(0, z)-k(x, 0)=0
$$

holds by Equation 3.3.

Remark 3.5. The condition $h(x, 0, z)=0$ in Equation 3.4 implies $h(x, y, z)=0$ as soon as one of $x, y, z$ is zero. To see this, use Equation 3.2 for $y=0$, giving $h(u, x, 0)=$ 0 and then Equation 3.2 with $x=0$. This shows that these cocycles are automatically assumed normalized in the sense of Definition 3.2.

Definition 3.6. A symmetric 3-cocycle is an abelian 3-cocycle $(h, c)$ such that

$$
c(x, y)=-c(y, x)
$$

for all $x, y \in G$. A symmetric 3-coboundary is the same as an abelian 3-coboundary.

Note that an abelian 3-coboundary is indeed always a symmetric 3-cocycle, as is seen from Equation 3.4. 
Definition 3.7. A quadratic form $q: G \rightarrow M$ is any map of sets such that $q(x)=$ $q(-x)$ and

$$
b(x, y):=q(x+y)-q(x)-q(y)
$$

is $\mathbb{Z}$-bilinear for all $x, y \in G$. This bilinear form is called the polarization form. Write $\operatorname{Quad}(G, M)$ for the set of all quadratic forms.

Theorem 3.8 (Eilenberg-Mac Lane). The group $H_{a b}^{3}(G, M)\left(\right.$ resp. $\left.H_{\text {sym }}^{3}(G, M)\right)$ of Definition 3.1 can be described as the set of abelian (resp. symmetric) 3-cocycles modulo abelian (resp. symmetric) 3-coboundaries in the sense of Definition 3.4 (resp. Definition 3.6). The map

$$
\begin{aligned}
\operatorname{tr}: H_{a b}^{3}(G, M) & \longrightarrow \operatorname{Quad}(G, M), \\
(h, c) & \longmapsto(x \mapsto c(x, x))
\end{aligned}
$$

is an isomorphism.

We refer to [ML52] for an overview. The paper [EML53] is essentially entirely devoted to making the type of translation underlying the above theorem.

\subsection{Joyal-Street classification}

We quickly summarize the Joyal-Street classification. We shall write $\mathcal{Q}$ uad for the 1-category (a) whose objects are triples $(G, M, q)$ with $G, M$ abelian groups and $q \in \operatorname{Quad}(G, M)$, and (b) morphisms $(G, M, q) \rightarrow\left(G^{\prime}, M^{\prime}, q^{\prime}\right)$ are pairs of group homomorphisms

$$
f: G \rightarrow G^{\prime} \quad \text { and } \quad g: M \rightarrow M^{\prime}
$$

such that the square

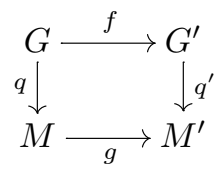

commutes. Write $\mathcal{B C G}$ for the 2-category (a) whose objects are braided categorical groups and (b) whose arrows are braided monoidal functors and (c) whose 2-arrows are braided monoidal natural equivalences of functors. For details on the precise definition we would perhaps recommend the nice and very careful treatment of CegarraKhmaladze [CK07, §6]. They treat the $G$-graded case, so for $G=1$ the trivial group, their description specializes to the concepts we use here. We write $H o(\mathcal{B C G})$ for what one could call the homotopy category of braided categorical groups (or 1-category truncation); its objects are braided categorical groups and morphisms are the equivalence classes of braided monoidal functors. As homotopy (or 2-equivalence) preserves composition, $\mathrm{Ho}(\mathcal{B C G})$ is a well-defined 1-category. There is a functor

$$
T: \mathrm{Ho}(\mathcal{B C G}) \longrightarrow \mathcal{Q u a d}, \quad(\mathrm{C}, \otimes) \mapsto\left(\pi_{0} \mathrm{C}, \pi_{1} \mathrm{C}, q\right),
$$

where $q$ is the quadratic form attached to the abelian 3-cohomology class encoding the associator and braiding, using Equation 3.6.

Theorem 3.9 (Joyal-Street). The functor $T$ has the following properties: 
1. It is well-defined.

2. It is essentially surjective.

3. It is conservative, i.e. $F:(\mathrm{C}, \otimes) \rightarrow\left(\mathrm{C}^{\prime}, \otimes^{\prime}\right)$ is a braided monoidal equivalence if and only if $T F$ is an isomorphism in $\mathcal{Q}$ uad.

4. It is full, i.e. any morphism of $\mathcal{Q}$ uad comes from a (homotopy class of) braided monoidal functor(s) of braided categorical groups.

See [JS93, Theorem 3.3 and Remark 3.3] or [CK07, page 435, again for $G=1$ and the category is called $\mathcal{H}_{1, a b}^{3}$ instead] or [JS86, §6-7]. Sính had earlier proven an analogous result in the case of symmetric braidings, i.e. Picard groupoids, [Sín75].

We can be a bit more precise: The essential surjectivity is proven by providing an explicit skeletal model with the given invariants:

Definition 3.10. Given abelian groups $G, M$ and an abelian 3-cocycle $(h, c)$, let $\mathcal{T}(G, M,(h, c))$ be the following braided categorical group:

1. objects are elements in $G$,

2. automorphisms of any object $X \in G$ are $\operatorname{Aut}(X):=M$,

3. there are no morphisms except for automorphisms, and their composition is addition in $M$,

4. the monoidal structure is

$$
(X \stackrel{f}{\longrightarrow} X) \otimes\left(X^{\prime} \stackrel{f^{\prime}}{\longrightarrow} X^{\prime}\right):=\left(X+X^{\prime} \stackrel{f+f^{\prime}}{\longrightarrow} X+X^{\prime}\right),
$$

where addition is just addition in $G$ (on objects) resp. in $M$ (for $f, f^{\prime}$ ),

5. the associator

$$
a_{X, Y, Z}: X \otimes(Y \otimes Z) \stackrel{\sim}{\longrightarrow}(X \otimes Y) \otimes Z
$$

is just the automorphism defined by $h(X, Y, Z) \in M$,

6. and analogously for the commutativity constraint and $c(X, Y)$.

As implied by Theorem 3.9 and the remark about how to prove essential surjectivity, any braided categorical group $(C, \otimes)$ is braided monoidal equivalent to the skeletal braided categorical group $\mathcal{T}\left(\pi_{0} \mathrm{C}, \pi_{1} \mathrm{C},(h, c)\right)$ for some $[(h, c)] \in H_{a b}^{3}\left(\pi_{0} \mathrm{C}, \pi_{1} \mathrm{C}\right)$, where $[(h, c)]$ is well-defined up to abelian 3 -coboundaries. Moreover, this detects braided monoidal equivalence: A braided monoidal functor $F:(C, \otimes) \rightarrow\left(C^{\prime}, \otimes^{\prime}\right)$ is an equivalence if and only if it induces isomorphisms on $\pi_{0}, \pi_{1}$ and under these the cohomology class gets identified. For Picard groupoids, these results were established earlier by Sính. The functor $T$ can also be used to describe various unstable homotopy types combinatorially by combining it with Equation 3.1. See [Bau91] for more on this.

Lemma 3.11. A skeletal braided categorical group is braided monoidal equivalent to a skeletal strictly associative one if and only if the cohomology class $[(h, c)]$ has an abelian 3 -cocycle representative with $h(x, y, z)=0$ for all $x, y, z \in G$.

Proof. Just use that any skeletal braided categorical group $\mathrm{C}$ is necessarily of the form $\mathcal{T}\left(\pi_{0} \mathrm{C}, \pi_{1} \mathrm{C},(h, c)\right)$ itself. 


\section{Polar forms}

\subsection{Polar quadratic forms}

Definition 4.1. We will call a quadratic form polar (or polar for $t$ ) if its polarization is of the shape

$$
b(x, y)=t(x, y)+t(y, x)
$$

for some bilinear form $t$ (which is not required to be symmetric or non-degenerate).

As we shall see in Lemma 4.12, this definition uniformly characterizes two somewhat different sources of examples in one.

Example 4.2. Suppose $t: G \times G \rightarrow M$ is a $\mathbb{Z}$-bilinear form, not necessarily symmetric. Then $q(x):=t(x, x)$ is a polar quadratic form by direct computation,

$$
b(x, y)=t(x, y)+t(y, x) .
$$

Example 4.3. Suppose $s: G \rightarrow M$ is any group homomorphism to an $\mathbb{F}_{2}$-vector space $M$. Then $q(x):=s(x)$ is a polar quadratic form because $b(x, y)=q(x+y)-q(x)-$ $q(y)=0$.

Example 4.4. Consider $q(x):=x^{2}$ as a map $\mathbb{Z} \rightarrow \mathbb{Z}$. This restricts to a well-defined map $\mathbb{Z} / 2 \rightarrow \mathbb{Z} / 4$ in view of

$$
(x+2 m)^{2}=x^{2}+4 m x+4 m^{2} \equiv x^{2} \bmod 4 .
$$

Thus, for $G:=\mathbb{Z} / 2$ and $M:=\mathbb{Z} / 4$ we obtain a quadratic form, $q(-x)=q(x)$, with the bilinear symmetric polarization $b(x, y):=2 x y$. We claim that this form is not polar. Assume it were. We must have $t: \mathbb{F}_{2} \times \mathbb{F}_{2} \rightarrow \mathbb{Z} / 4 \mathbb{Z}$ and $t(x, y)=x y n$ for some $n \in \mathbb{Z}$ by bilinearity, and thus without loss of generality $n=1$ as we need $b(x, y)=$ $t(x, y)+t(y, x)$. However, $(x, y) \mapsto x y$ is not bilinear as a map $\mathbb{F}_{2} \times \mathbb{F}_{2} \rightarrow \mathbb{Z} / 4 \mathbb{Z}$ : We have

$$
0=c(0,1)=c(1+1,1) \neq c(1,1)+c(1,1)=2 \quad \text { in } \quad \mathbb{Z} / 4 .
$$

On the other hand, define

$$
h(x, y, z):= \begin{cases}2 & \text { for } x=y=z=1 \\ 0 & \text { else }\end{cases}
$$

and $c(x, y):=x y$, which is just

$$
c(x, y):= \begin{cases}1 & \text { for } x=y=z=1 \\ 0 & \text { else. }\end{cases}
$$

Then $(h, c)$ is an abelian 3 -cocycle. We repeat that $c$ is not bilinear, contrary to what the trained eye might misconceive.

Lemma 4.5. An arbitrary map of sets $q: G \rightarrow M$ is quadratic if and only if

$$
\begin{aligned}
q(-x) & =q(x), \\
q(x+y+z)+q(x)+q(y)+q(z) & =q(y+z)+q(z+x)+q(x+y)
\end{aligned}
$$

both hold for all $x, y, z \in G$. 
Proof. We demand $q(-x)=q(x)$ in both situations, so we only need to show that Equation 4.1 is equivalent to the $\mathbb{Z}$-bilinearity of $b(x, y)$ in Equation 3.5. Since the latter is visibly symmetric, it suffices to check linearity in the first variable, that is, the vanishing of

$$
b(x+y, z)-b(x, z)-b(y, z)
$$

for all $x, y, z$. When unravelling each $b(-,-)$ using Equation 3.5, we obtain an expression which is zero if and only if Equation 4.1 holds.

Lemma 4.6. If $(h, c)$ is an abelian 3-cocycle, we have

$$
\sum_{\sigma \in S_{3}} \operatorname{sgn}(\sigma) h\left(x_{\sigma(1)}, x_{\sigma(2)}, x_{\sigma(3)}\right)=0,
$$

i.e. the alternating sum over all permutations of the arguments is zero.

Proof. In Identity A bring all terms relying on $c$ on the left side, giving

$$
c(x, y+z)-c(x, y)-c(x, z)=-h(x, y, z)+h(y, x, z)-h(y, z, x) .
$$

The left side of the equation is invariant under swapping $y$ and $z$, and thus so must be the right side. Thus, their difference

$$
-h(x, y, z)+h(y, x, z)-h(y, z, x)+h(x, z, y)-h(z, x, y)+h(z, y, x)=0
$$

must be zero. But this is what we had to prove.

Lemma 4.7. If $(h, c)$ is an abelian 3 -cocycle, then $W(x, y):=c(x, y)+c(y, x)$ is $\mathbb{Z}$ bilinear and symmetric.

Proof. Since $W$ is symmetric in $x, y$, it suffices to check linearity in $x$. We find

$$
\begin{aligned}
W(x+z, y)-W(x, y)-W(z, y)= & c(x+z, y)+c(y, x+z) \\
& -c(x, y)-c(y, x)-c(z, y)-c(y, z)
\end{aligned}
$$

and evaluating $c(x+z, y)$ using Identity A' (and observing a number of cancellations), we obtain

$$
=h(x, z, y)-h(x, y, z)+h(y, x, z)+c(y, x+z)-c(y, x)-c(y, z) .
$$

Next, unravel $c(y, x+z)$ using Identity A, giving

$$
=h(x, z, y)-h(x, y, z)+h(y, x, z)-h(y, x, z)+h(x, y, z)-h(x, z, y)=0,
$$

which vanishes (all terms appear twice, but with opposite signs).

Lemma 4.8. If $(h, c)$ is an abelian 3-cocycle, then we have

$$
c(y+z, y+z)-c(y, y)-c(z, z)=W(y, z)
$$

for all $y, z$ and $W$ as in the previous lemma. 
Proof. We again use Identity $\mathrm{A}$, in the form

$$
c(x, y+z)-c(x, y)-c(x, z)=-h(x, y, z)+h(y, x, z)-h(y, z, x) .
$$

Now we plug in $x:=y+z$. This yields

$$
\begin{array}{r}
c(y+z, y+z)-c(y+z, y)-c(y+z, z) \\
\quad=-h(y+z, y, z)+h(y, y+z, z)-h(y, z, y+z)
\end{array}
$$

and now use Identity A' to unravel $c(y+z, y)$ and $c(y+z, z)$. We get

$$
\begin{aligned}
c(y+z, y+z) & -c(z, y)-c(y, y)-c(y, z)-c(z, z) \\
= & -h(y+z, y, z)+h(y, y+z, z)-h(y, z, y+z)+h(z, y, z)+h(y, z, y) .
\end{aligned}
$$

Recall the group 3-cocycle condition, Equation 3.2, but plug in $u:=y$ and $x:=z$. Then the cocycle condition tells us that the right side of this equation vanishes. This proves our claim.

Lemma 4.9. If $(h, c)$ is an abelian 3-cocycle, then $q(x):=c(x, x)$ is a quadratic form.

This quadratic form is called the trace and underlies the map of Equation 1.1. The above lemma is of course due to Eilenberg and Mac Lane, and quite old, but since almost all texts just refer to this verification as an Exercise, we felt we should spell it out, if only to provide a reference.

Proof. (Step 1) We compute, just by unravelling $q$ in terms of $c$ and using Lemma 4.8 three times, that $q(x+y)+q(y+z)+q(x+z)$ is

$$
\begin{aligned}
& =c(x+y, x+y)+c(y+z, y+z)+c(x+z, x+z) \\
& =2 c(x, x)+2 c(y, y)+2 c(z, z)+W(x, y)+W(y, z)+W(x, z) .
\end{aligned}
$$

Next, use Lemma 4.8 but plug in $x+y$ instead of $y$, giving

$$
c(x+y+z, x+y+z)-c(x+y, x+y)-c(z, z)=W(x+y, z) .
$$

Apply Lemma 4.8 to unravel $c(x+y, x+y)$ and use the bilinearity of $W$ (Lemma 4.7), giving

$$
c(x+y+z, x+y+z)-c(x, x)-c(y, y)-c(z, z)=W(x, y)+W(x, z)+W(y, z) .
$$

Now, we plug in Equation 4.2 for the $W$-terms in the above equation, giving

$$
q(x+y+z)+q(x)+q(y)+q(z)=q(x+y)+q(y+z)+q(x+z) .
$$

Thus, by Lemma 4.5 , we are done once we show that $q(x)=q(-x)$.

(Step 2) To this end, use Equation 4.3 with $y:=-x, z:=x$. We get

$$
3 q(x)+q(-x)=2 q(0)+q(2 x) .
$$

Lemma 4.8 with all variables zero shows $-q(0)=W(0,0)=0$ by the bilinearity of $W$. Next, use Lemma 4.8 with $y=z$ and call the variable $x$. We get $q(2 x)-2 q(x)=$ $W(x, x)$. Combine all three formulas to obtain $q(x)+q(-x)=W(x, x)$, but unravelling $W$ using its definition yields $2 q(x)$. Thus, we obtain $q(-x)=q(x)$, as desired.

The following goes back to Whitehead [Whi50]. 
Lemma 4.10. There is a commutative diagram

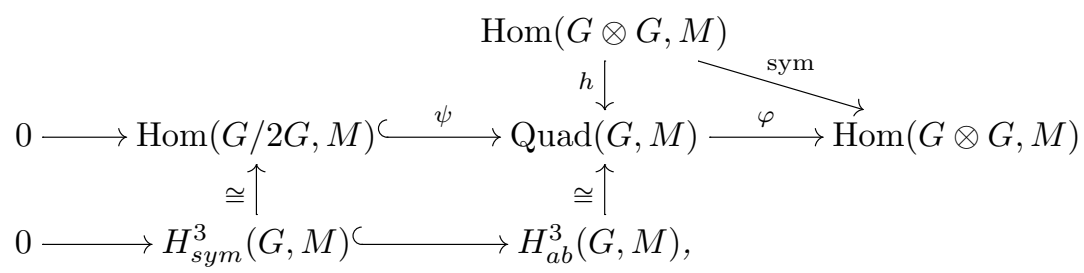

where $\psi$ sends a map to itself and $\varphi$ sends $q$ to its polarization, Equation 3.5. The downward arrow $h$ sends a bilinear map $B$ to $q(x):=B(x, x)$, and the diagonal arrow is the symmetrization map

$$
b(x, y):=B(x, y)+B(y, x) .
$$

The middle row in the diagram is exact. The upward arrows send $(h, c)$ to $x \mapsto c(x, x)$.

Proof. The commutativity of the upper triangle is a one line computation. We check the exactness of the middle row: If $f: G / 2 \rightarrow M$ is any $\mathbb{Z}$-linear map and $b=\varphi(f)$, then we clearly have $b(x, y)=0$ in Equation 3.5 by linearity and $f(a)=f(-a)$ holds since all elements in $G / 2$ are 2 -torsion, so $\psi$ is well-defined and injective (see also Example 4.3). If $\varphi$ sends a quadratic form $q$ to zero, we have $q(x+y)=q(x)+q(y)$, so it follows that $q$ is $\mathbb{Z}$-linear. Moreover, $q(x)=q(-x)=-q(x)$ forces that $2 q(x)=0$ for all $x$, but this is $q(2 x)$. Hence, $2 G$ lies in the kernel and by the universal property of cokernels, we get a unique map $f: G / 2 G \rightarrow M$, giving exactness in the middle. The inclusion $H_{\text {sym }}^{3}(G, M) \subseteq H_{a b}^{3}(G, M)$ follows from the fact that while symmetric 3 -cocycles have the additional constraint

$$
c(x, y)=-c(y, x),
$$

the sets of symmetric vs. abelian 3-coboundaries are the same (Definition 3.6). The lower square commutes because the upward arrows are the same map, just restricted to a subgroup, and on all of $H_{a b}^{3}(G, M)$ its values are quadratic forms by Lemma 4.9. Further, Equation 4.5 implies $2 c(x, x)=0$, so the image of the subgroup $H_{\text {sym }}^{3}(G, M)$ lands in those quadratic forms such that the polarization is zero, so it factors over $\operatorname{Hom}(G / 2 G, M)$ by the exactness of the middle row. The upward arrows being isomorphisms follows from Theorem 3.8.

Lemma 4.11. Suppose $G$ is a free abelian group, $M$ arbitrary. Then every quadratic form $q: G \rightarrow M$ is polar.

The following argument is vaguely analogous to [DGNO10, Lemma D.1].

Proof. Pick a basis $\left(e_{i}\right)_{i \in I}$ (for some index set $I$ ) of $G$ as a free $\mathbb{Z}$-module. Write $b(x, y)$ for the polarization of $q$, as in Equation 3.5. Pick an arbitrary total order on the set $I$. Define

$$
t\left(e_{i}, e_{j}\right):= \begin{cases}b\left(e_{i}, e_{j}\right) & \text { if } i<j, \\ q\left(e_{i}\right) & \text { if } i=j, \\ 0 & \text { if } i>j .\end{cases}
$$

Then if $i \neq j$ we have $t\left(e_{i}, e_{j}\right)+t\left(e_{j}, e_{i}\right)=b\left(e_{i}, e_{j}\right)$ and for $i=j$ we get $t\left(e_{i}, e_{i}\right)+$ $t\left(e_{i}, e_{i}\right)=2 q\left(e_{i}\right)$, but we also have $b\left(e_{i}, e_{i}\right)=q\left(2 e_{i}\right)-2 q\left(e_{i}\right)=2 q\left(e_{i}\right)$ since $q(2 x)=$ 
$4 q(x)$ for all $x \in G$ by the same computation as in Equation 4.4. Thus, by $\mathbb{Z}$-linear extension and since $\left(e_{i}\right)_{i \in I}$ is a basis, we conclude that $t(x, y)+t(y, x)=b(x, y)$ holds for all $x, y \in G$.

For an abelian group $M$ we write ${ }_{n} M=\operatorname{ker}(M \stackrel{\cdot n}{\longrightarrow} M)$.

Lemma 4.12. Let $t: G \times G \rightarrow M$ be a bilinear map. A quadratic form $q: G \rightarrow M$

1. is polar for this $t$, if and only if

2. there exists some $\bar{q} \in \operatorname{Hom}_{\mathbb{F}_{2}}\left(G / 2 G,{ }_{2} M\right)$ such that $q(x)=t(x, x)+\bar{q}(x)$ holds for all $x \in G$.

Proof. Suppose $q$ is polar for the given $t$. As usual, let $b(x, y):=q(x+y)-q(x)-$ $q(y)$ be its polarization form, and by assumption we have $b(x, y)=t(x, y)+t(y, x)$. Define $\bar{q}(x):=q(x)-t(x, x)$ for $x \in G$. We claim that $\bar{q}$ is a quadratic form. Indeed,

$$
\bar{q}(-x)=q(-x)-t(-x,-x)=q(x)-t(x, x)=\bar{q}(x)
$$

since $q$ is quadratic and $t$ bilinear. The polarization of $\bar{q}$ is

$$
\begin{aligned}
\bar{b}(x, y) & =\bar{q}(x+y)-\bar{q}(x)-\bar{q}(y) \\
& =q(x+y)-t(x+y, x+y)-q(x)+t(x, x)-q(y)+t(y, y) \\
& =b(x, y)-(t(x, y)+t(y, x))=0 .
\end{aligned}
$$

We see that this polarization is (trivially) $\mathbb{Z}$-bilinear, completing the verification that $\bar{q}$ is quadratic, but also proving that $\bar{q} \in \operatorname{Hom}_{\mathbb{F}_{2}}\left(G / 2 G,{ }_{2} M\right)$ by the exactness of middle row in Lemma 4.10. Conversely, suppose we know that $\bar{q}$ exists. Then $q$ being polar for $t$ amounts to combining Example 4.2 and Example 4.3.

\subsection{Proof of the main results}

Theorem 4.13. Let $G, M$ be abelian groups. Let $\left(\beta_{i}\right)_{i \in I}$ be a basis of the $\mathbb{F}_{2}$-vector space $G / 2 G$. Let $q \in \operatorname{Quad}(G, M)$ be a polar quadratic form. Then it can be written as

$$
q(x)=t(x, x)+\bar{q}(x)
$$

(by Lemma 4.12) for $t: G \times G \rightarrow M$ bilinear and $\bar{q} \in \operatorname{Hom}_{\mathbb{F}_{2}}\left(G / 2 G,{ }_{2} M\right)$. Define an abelian 3-cocycle

$$
\begin{aligned}
h(x, y, z) & :=0, \\
c(x, y) & :=t(x, y)+\sum_{i \in I} \bar{x}_{i} \cdot \bar{y}_{i} \cdot \bar{q}\left(\beta_{i}\right),
\end{aligned}
$$

where $\bar{x}, \bar{y}$ are the vectors we get under the quotient map $G \rightarrow G / 2 G$, spelled out with respect to the basis $\left(\beta_{i}\right)$. This means that $\bar{x}_{i}, \bar{y}_{i} \in \mathbb{F}_{2}$. Then the trace of $(h, c)$ is just the quadratic form $q$. That is, we have found a preimage under

$$
H_{a b}^{3}(G, M) \stackrel{\sim}{\longrightarrow} \operatorname{Quad}(G, M) .
$$

This preimage is independent of the choice of the basis $\left(\beta_{i}\right)$. 
Proof. (Step 1) Note that we can write the polarization of $q$ as

$$
b(x, y)=q(x+y)-q(x)-q(y)=t(x, y)+t(y, x)
$$

for the bilinear form $t: G \times G \rightarrow M$ in the statement of the theorem by Lemma 4.12. Next, we check that $(h, c)$ is an abelian 3-cocycle. Indeed, having $h(x, y, z)=0$, Identity A and A' simplify to the condition that $c: G \times G \rightarrow M$ is supposed to be $\mathbb{Z}$ bilinear, so this is all we have to show. Since $t$ is $\mathbb{Z}$-bilinear, it suffices to prove that

$$
c^{\prime}(x, y):=\sum_{i \in I} \bar{x}_{i} \cdot \bar{y}_{i} \cdot \bar{q}\left(\beta_{i}\right)
$$

is $\mathbb{Z}$-bilinear. However, this is clear since each $\bar{q}\left(\beta_{i}\right) \in{ }_{2} M$ is a 2-torsion element; it factors over $G / 2 G \times G / 2 G \rightarrow{ }_{2} M$, where it is a bilinear form on $\mathbb{F}_{2}$-vector spaces.

(Step 2) Now that we know that $(h, c)$ is an abelian 3-cocycle, we need to check that its trace is $q$. Let us denote its trace by $Q$, i.e. $Q(x):=c(x, x)$. We obtain the explicit formula

$$
Q(x)=t(x, x)+\sum_{i \in I} \bar{x}_{i}^{2} \cdot \bar{q}\left(\beta_{i}\right)=t(x, x)+\sum_{i \in I} \bar{x}_{i} \cdot \bar{q}\left(\beta_{i}\right),
$$

where we exploit that the values of $\bar{q}$ lie in the $\mathbb{F}_{2}$-vector space ${ }_{2} M$, and $n^{2} \equiv n$ in $\mathbb{F}_{2}$ for all integers. The polarization of $Q$ is

$$
B(x, y)=Q(x+y)-Q(x)-Q(y)=t(x, y)+t(y, x)=b(x, y) .
$$

However, this is also the polarization of $q$, see Equation 4.7. Thus, by the exactness of middle row in Lemma 4.10 we deduce that the quadratic form $Q-q$ comes from $\operatorname{Hom}_{\mathbb{F}_{2}}\left(G / 2 G,{ }_{2} M\right)$. In particular, $Q-q$ is a linear map on the $\mathbb{F}_{2}$-vector space $G / 2 G$. In order to show that it is zero, it suffices to verify that it is zero on the basis vectors $\beta_{j}$ with $j \in I$. We compute

$$
(Q-q)(x)=t(x, x)-q(x)+\sum_{i \in I} \bar{x}_{i} \cdot\left(q\left(\beta_{i}\right)-t\left(\beta_{i}, \beta_{i}\right)\right) .
$$

So for $x:=\beta_{j}$ we have $\bar{x}_{i}=\delta_{i=j}$ (Kronecker delta) and thus

$$
(Q-q)\left(\beta_{j}\right)=t\left(\beta_{j}, \beta_{j}\right)-q\left(\beta_{j}\right)+q\left(\beta_{j}\right)-t\left(\beta_{j}, \beta_{j}\right)=0 .
$$

As this also vanishes, Lemma 4.10 implies that $Q-q$ is the zero quadratic form, i.e. $Q=q$. Finally, our class $(h, c)$ is independent of the choice of the basis $\left(\beta_{i}\right)_{i \in I}$ since by the Eilenberg-Mac Lane theorem (Theorem 3.8) the trace map in Equation 4.6 is bijective.

Corollary 4.14. A quadratic form $q$ is polar if and only if there exists a bilinear form $S: G \times G \rightarrow M$ such that $q(x)=S(x, x)$.

Proof. If it is polar, then by Theorem 4.13 we have $q(x)=c(x, x)$ and $c$ is bilinear, so we can take $S(x, y):=c(x, y)$. The converse is immediate, see Example 4.2.

Theorem 4.15. A braided categorical group $(\mathrm{C}, \otimes)$ is braided monoidal equivalent to one which is simultaneously strictly associative and skeletal if and only if its quadratic form is polar. 
Proof. We find a braided monoidal equivalence $(\mathrm{C}, \otimes) \simeq \mathcal{T}(G, M,(h, c))$ for $G:=\pi_{0} \mathrm{C}$, $M:=\pi_{1} \mathrm{C}$, and $(h, c) \in H_{a b}^{3}(G, M)$ by the properties of the functor $T$ of Theorem 3.9. Being skeletal, the triviality of the associator means that $h(x, y, z)=0$ for all $x, y, z$, Lemma 3.11. Using this, Identity A and A' simplify to say that $c: G \times G \rightarrow M$ is bilinear. Let $q(x):=c(x, x)$ be its trace, which is quadratic by Lemma 4.9. For the polarization of $q$ we compute

$$
b(x, y)=c(x+y, x+y)-c(x, x)-c(y, y)=c(x, y)+c(y, x) .
$$

Thus, $q$ is polar, because we may use $t(x, y):=c(x, y)$ in Definition 4.1. Conversely, suppose $(h, c)$ under the Eilenberg-Mac Lane isomorphism of Equation 1.1 gets sent to a polar quadratic form. Then we may apply Theorem 4.13 and it produces a cohomologous abelian 3 -cocycle representative of the shape $\left(0, c^{\prime}\right)$, so reversely by Lemma 3.11 we get a braided monoidal equivalence

$$
(\mathrm{C}, \otimes) \simeq \mathcal{T}\left(G, M,\left(0, c^{\prime}\right)\right),
$$

from Theorem 3.9, but the right side is both skeletal and strictly associative.

Theorem 4.16. For every braided categorical group $(\mathrm{C}, \otimes)$, there exists an essentially surjective and faithful (but typically not full) braided monoidal functor from another braided categorical group

$$
(\widehat{\mathrm{C}}, \otimes) \longrightarrow(\mathrm{C}, \otimes),
$$

surjective on $\pi_{0}$, and an isomorphism on $\pi_{1}$, such that $(\widehat{\mathrm{C}}, \otimes)$ is simultaneously strictly associative and skeletal. We call $(\widehat{\mathrm{C}}, \otimes)$ a polar cover of $(\mathrm{C}, \otimes)$.

Proof. By the Joyal-Street classification (Theorem 3.9), there exists an equivalence

$$
F:(\mathrm{C}, \otimes) \stackrel{\sim}{\rightarrow} \mathcal{T}\left(\pi_{0} \mathrm{C}, \pi_{1} \mathrm{C},(h, c)\right)
$$

for $(h, c)$ an abelian 3 -cocycle representing the relevant cohomology class. Let $c: P \rightarrow$ $\pi_{0} \mathrm{C}$ be a surjective homomorphism from a free abelian group $P$ (e.g., pick a projective cover of $\pi_{0} C$ as a $\mathbb{Z}$-module). We get a commutative square

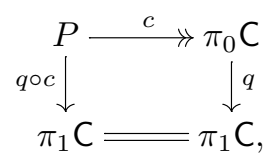

describing a morphism $\left(P, \pi_{1} \mathrm{C}, q \circ c\right) \rightarrow\left(\pi_{0} \mathrm{C}, \pi_{1} \mathrm{C}, q\right)$ in the category $\mathcal{Q}$ uad of $\S 3.2$. Note that $q \circ c$ is indeed again a quadratic form since $c$ is $\mathbb{Z}$-linear. As the functor $T$ of Theorem 3.9 is full and essentially surjective, and the skeletal categorical groups $\mathcal{T}(-,-,-)$ are concrete representatives for the essential surjectivity, we obtain some braided monoidal functor

$$
G: \mathcal{T}\left(P, \pi_{1} \mathrm{C},\left(h^{\prime}, c^{\prime}\right)\right) \longrightarrow \mathcal{T}\left(\pi_{0} \mathrm{C}, \pi_{1} \mathrm{C},(h, c)\right),
$$

where $\left(h^{\prime}, c^{\prime}\right)$ is an abelian 3-cocycle corresponding to the lifted quadratic form $q \circ c$ under the Eilenberg-Mac Lane isomorphism of Equation 1.1. Next, by Lemma 4.11 since $P$ is free abelian, the quadratic form $q \circ c$ is necessarily polar and thus has a representative of the shape $\left(0, c^{\prime \prime}\right)$ within its cohomology class in $H_{a b}^{3}\left(P, \pi_{1} \mathrm{C}\right)$ by 
Theorem 4.13. Hence, we can pre-compose $G$ with a braided monoidal equivalence

$$
\mathcal{T}\left(P, \pi_{1} \mathrm{C},\left(0, c^{\prime \prime}\right)\right) \stackrel{\sim}{\longrightarrow} \mathcal{T}\left(P, \pi_{1} \mathrm{C},\left(h^{\prime}, c^{\prime}\right)\right) \longrightarrow \mathcal{T}\left(\pi_{0} \mathrm{C}, \pi_{1} \mathrm{C},(h, c)\right)
$$

and as explained in Lemma 3.11 the braided categorical group $\mathcal{T}\left(P, \pi_{1} \mathrm{C},\left(0, c^{\prime \prime}\right)\right)$ is both skeletal and strictly associative. Call it $(\widehat{\mathrm{C}}, \otimes)$. Using the above composition of functors, we get the braided monoidal functor of Equation 4.8; the surjection on the level of $\pi_{0}$ is the map $c$, and the isomorphy on the level of $\pi_{1}$ is clear. As the map on the level of $\pi_{0}$ is surjective, the functor is essentially surjective. On the level of morphisms only two things can happen: Morphisms are $\pi_{1} \mathrm{C}$ if the objects are isomorphic, or vacuous otherwise. It follows that the functor is faithful, but not necessarily full since non-isomorphic objects may become isomorphic, namely when two distinct elements of $P$ map to the same element in $\pi_{0} \mathrm{C}$.

\section{Appendix A. Strictifying the universal determinant}

Finally, we explain a consequence of our results to Deligne's universal determinant functor of an exact category. Allowing ourselves an anachronistic interpretation, the idea of the universal determinant functor is as follows:

Take the truncation of the $K$-theory spectrum $K(\mathrm{C})$ of an exact category $\mathrm{C}$ to its stable homotopy $[0,1]$-type. We get a map $K(\mathrm{C}) \rightarrow \tau_{\leqslant 1} K(\mathrm{C})$ and using a stable variant of Equation 3.1 (e.g., concretely [JO12] or [Pat12, §5]), the stable homotopy $[0,1]$-type $\tau_{\leqslant 1} K(\mathrm{C})$ can be modelled as a Picard groupoid. Deligne has observed in his paper [Del87, §4.2] that this map and the relevant Picard groupoid can equivalently be described as the target of the universal determinant functor defined on the category $C$, giving a formulation which is a priori independent of any algebraic $K$-theory. This yields a connection to the far less homotopically defined determinant functors in terms of top exterior powers of vector bundles, as they would occur in algebraic geometry (e.g., the determinant line bundle on moduli spaces of vector bundles on curves, as just one possible application).

Now, being a Picard groupoid, it already follows from the result of Johnson-Osorno [JO12] that $\tau_{\leqslant 1} K(\mathrm{C})$ can be made skeletal and strictly associative. In this appendix we explain how to pin down the relevant symmetry constraint, using the formula from our Theorem 4.13. It will also follow readily that besides the target, the entire universal determinant functor can be strictified.

We have not found this fact recorded anywhere in the literature.

In detail: Suppose $C$ is an exact category with a fixed zero object. Deligne constructs the Picard groupoid of virtual objects $V(\mathrm{C})$ : Let $Q C$ be the Quillen $Q$ construction of C; see for example [Wei13, Chapter IV, §6] for a precise definition. Write $N_{\bullet} Q C$ for its nerve. As $Q C$ has the same objects as C itself, the fixed zero object pins down a 0 -simplex of $N_{\bullet} Q \mathrm{C}$, rendering the latter a pointed simplicial set.

Set up a new category $V(\mathrm{C})$

1. whose objects are closed loops in the space $N_{\bullet} Q \mathrm{C}$ around the base point, and

2. whose morphisms are based homotopy classes of homotopies between loops.

The composition of morphisms is defined as the composition of homotopies. The associativity law for composition then holds (and only holds) because morphisms are only taken modulo their based homotopy class. 
One checks that the above makes $V(\mathrm{C})$ a groupoid. A monoidal structure on $V(\mathrm{C})$, i.e. a suitable bifunctor $\otimes: V(\mathrm{C}) \times V(\mathrm{C}) \rightarrow V(\mathrm{C})$ is defined as the composition of loops on the level of objects. This bifunctor can be promoted to a symmetric monoidal structure. We refer to [Del87, §4.2] for further details.

In [Del87, §4.3] Deligne gives a second construction of $V(\mathrm{C})$. He first sets up the concept of a determinant functor. Given any exact category $C$, we write $C^{\times}$for the same category, except that we only keep isomorphisms as morphisms (this is sometimes called the maximal inner groupoid or group core). This is a groupoid.

Definition A.1 ([Del87, §4.3]). Let $\mathrm{C}$ be an exact category and let $(\mathrm{P}, \otimes)$ be a Picard groupoid. A determinant functor on $\mathrm{C}$ is a functor $\mathcal{D}: \mathrm{C}^{\times} \rightarrow \mathrm{P}$ along with the following extra structure and axioms:

1. For any exact sequence $\Sigma: G^{\prime} \hookrightarrow G \rightarrow G^{\prime \prime}$ in $\mathrm{C}$, we are given an isomorphism

$$
\mathcal{D}(\Sigma): \mathcal{D}(G) \stackrel{\sim}{\longrightarrow} \mathcal{D}\left(G^{\prime}\right) \underset{\mathrm{P}}{\otimes} \mathcal{D}\left(G^{\prime \prime}\right)
$$

in $\mathrm{P}$. This isomorphism is required to be functorial in morphisms of exact sequences.

2. For every zero object $Z$ of $\mathrm{C}$, we are given an isomorphism $z: \mathcal{D}(Z) \stackrel{\sim}{\rightarrow} \mathbf{1}_{\mathrm{P}}$ to the unit object of the Picard groupoid.

3. Suppose $f: G \rightarrow G^{\prime}$ is an isomorphism in C. We write

$$
\Sigma_{l}: 0 \hookrightarrow G \rightarrow G^{\prime} \quad \text { and } \quad \Sigma_{r}: G \hookrightarrow G^{\prime} \rightarrow 0
$$

for the depicted exact sequences. We demand that the composition

$$
\mathcal{D}(G) \underset{\mathcal{D}\left(\Sigma_{l}\right)}{\stackrel{\sim}{\longrightarrow}} \mathcal{D}(0) \underset{\mathrm{P}}{\otimes} \mathcal{D}\left(G^{\prime}\right) \underset{z \otimes 1}{\stackrel{\sim}{\longrightarrow}} \mathbf{1}_{\mathrm{P}} \underset{\mathrm{P}}{\otimes} \mathcal{D}\left(G^{\prime}\right) \underset{\mathrm{P}}{\stackrel{\sim}{\longrightarrow}} \mathcal{D}\left(G^{\prime}\right)
$$

and the natural map $\mathcal{D}(f): \mathcal{D}(G) \stackrel{\sim}{\rightarrow} \mathcal{D}\left(G^{\prime}\right)$ agree. Further, require that $\mathcal{D}\left(f^{-1}\right)$ agrees with a variant of Equation A.1 using $\Sigma_{r}$ instead of $\Sigma_{l}$.

4. If a two-step filtration $G_{1} \hookrightarrow G_{2} \hookrightarrow G_{3}$ is given, we demand that the diagram

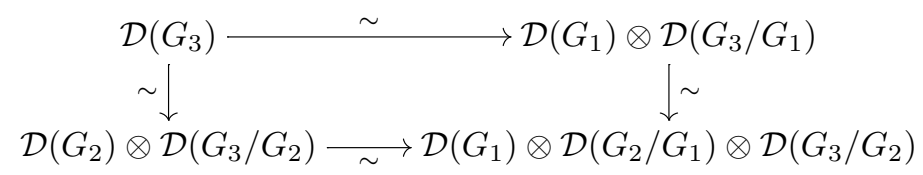

commutes.

5. Given objects $G, G^{\prime} \in \mathrm{C}$ consider the exact sequences

$$
\Sigma_{1}: G \hookrightarrow G \oplus G^{\prime} \rightarrow G^{\prime} \quad \text { and } \quad \Sigma_{2}: G^{\prime} \hookrightarrow G \oplus G^{\prime} \rightarrow G
$$

with the natural inclusion and projection morphisms. Then the diagram

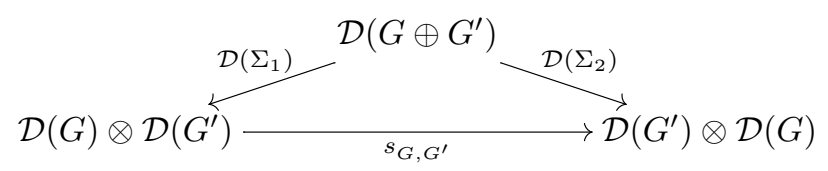

commutes, where $s_{G, G^{\prime}}$ denotes the symmetry constraint of P. 
At the end of $[$ Del87, $\S 4.3]$ Deligne now considers the category $\operatorname{det}(\mathrm{C}, \mathrm{P})$ of determinant functors, i.e.

1. objects are determinant functors in the sense of the above definition, and

2. morphisms are natural transformations of determinant functors.

Details for this are spelled out in [Bre11, §2.3], especially a full description of a morphism of determinant functors is [Bre11, Definition 2.5]. We also took over his notation $\operatorname{det}(\mathrm{C}, \mathrm{P})$ for this category.

Definition A.2. A determinant functor $\mathcal{D}: C^{\times} \longrightarrow P$ is called universal if for every given Picard groupoid $\mathrm{P}^{\prime}$ the functor

$$
\operatorname{Hom}^{\otimes}\left(\mathrm{P}, \mathrm{P}^{\prime}\right) \longrightarrow \operatorname{det}\left(\mathrm{C}, \mathrm{P}^{\prime}\right), \quad \varphi \mapsto \varphi \circ \mathcal{D}
$$

is an equivalence of categories.

This is already in Deligne [Del87, §4.3], but perhaps a little more detailed in [Bre11, §4.1]. Deligne then argues that a universal determinant functor exists and can be constructed using $V(\mathrm{C})$. To set it up, recall that the $Q$-construction category $Q C$ has the same objects as C, and for every admissible monic (resp. epic) $f$ in C, there are arrows $f_{!}$(resp. $f^{!}$) in $Q C$; see for example [Qui73, §2] or [Sri96, Chapter 6]. Let 0 be the fixed zero object of $C$. We use the notation

$$
0_{!}^{A}=(0 \nleftarrow 0 \hookrightarrow A) \quad \text { and } \quad 0_{A}^{!}=(0 \leftarrow A \hookrightarrow A),
$$

using the canonical arrows coming from the fact that 0 is both initial and final in $\mathrm{C}$. For every object $X \in \mathrm{C}$ one considers the diagram

$$
0 \stackrel{0_{!}^{X}}{\longrightarrow} X \stackrel{0_{X}^{!}}{\longleftarrow} 0
$$

showing that $\left(0_{!}^{A}\right)^{-1} \circ 0_{A}^{!}$is a closed loop around the base point in the nerve of $Q \mathrm{C}$. We denote it by $[X]$. We can now formulate Deligne's fundamental result. Recall that defining a morphism in $V(\mathrm{C})$ can be done by pinning down a homotopy of loops.

Theorem A.3 (Deligne, [Del87, §4.4-4.5]). Let C be an exact category with a fixed zero object 0 . Then $V(\mathrm{C})$ is a Picard groupoid. Define a functor $\mathcal{D}: \mathrm{C}^{\times} \rightarrow V(\mathrm{C})$ by

$$
X \mapsto[X]
$$

on objects, i.e. the loop of Equation A.4. Isomorphisms $\varphi: X \rightarrow Y$ give rise to a homotopy of loops

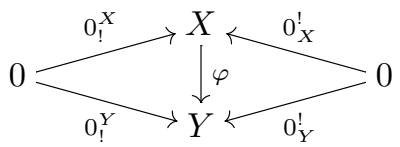

and this defines the functor on morphisms. To any exact sequence

$$
\Sigma: \quad A \stackrel{\alpha}{\rightarrow} B \stackrel{\beta}{\rightarrow} C
$$


attach the homotopy $\mathcal{D}(\Sigma)$

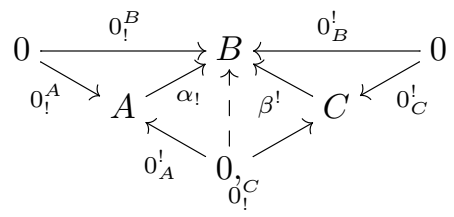

which are four 2-simplices giving a homotopy between the required loops. The dashed arrow is $(0 \leftarrow A \stackrel{\alpha}{\hookrightarrow} B)$ in $Q C$. Then $\mathcal{D}$ is a universal determinant functor.

Usually, although Definition A.2 is a little more subtle in its formulation, this is (very reasonably) simply called the universal determinant functor. Next, we shall apply the strictification methods of $\S 4$ to Deligne's constructions. First of all, $V(\mathrm{C})$ is a Picard groupoid, i.e. a braided categorical group whose braiding is symmetric. We apply Joyal-Street's skeletalization, giving a braided (symmetric) monoidal equivalence

$$
V(\mathrm{C}) \stackrel{\sim}{\longrightarrow} \mathcal{T}\left(\pi_{0} V(\mathrm{C}), \pi_{1} V(\mathrm{C}),(h, c)\right),
$$

where $[(h, c)]$ is the abelian 3-cohomology class in $H_{a b}^{3}\left(\pi_{0} V(\mathrm{C}), \pi_{1} V(\mathrm{C})\right)$ encoding the braiding and associativity constraint. Since we have canonical isomorphisms

$$
\pi_{0} V(\mathrm{C}) \cong K_{0}(\mathrm{C}) \quad \text { and } \quad \pi_{1} V(\mathrm{C}) \cong K_{1}(\mathrm{C}),
$$

using that the virtual objects arise as the truncation of the $K$-theory spectrum to its stable $[0,1]$-type, we understand these groups. Moreover, since the braiding is symmetric, this cocycle lies in the subgroup $H_{\text {sym }}^{3} \subseteq H_{a b}^{3}$, as in Lemma 4.10.

It follows from the exact middle row in Lemma 4.10 that the polarization form of the quadratic form $q \in \operatorname{Quad}\left(K_{0}(\mathrm{C}), K_{1}(\mathrm{C})\right)$ is zero. In particular, $q$ is polar; just pick $t(x, y):=0$ in Definition 4.1. Now apply Theorem 4.13. Let $\left(\beta_{i}\right)_{i \in I}$ be a basis of the $\mathbb{F}_{2}$-vector space $K_{0}(\mathrm{C}) / 2 K_{0}(\mathrm{C})$. We have $\bar{q}=q$ (in the notation of the cited theorem) since $t$ vanishes, so the symmetric 3 -cocycle

$$
h(x, y, z):=0 \quad c(x, y):=\sum_{i \in I} \bar{x}_{i} \cdot \bar{y}_{i} \cdot q\left(\beta_{i}\right)
$$

is a representative of the symmetric cohomology class of $[(h, c)]$. Without loss of generality, we may assume that this is the representative we had started with. We next compute the $q\left(\beta_{i}\right)$ in terms of the original exact category C. Returning to Lemma 4.10, we see that $q$ is an $\mathbb{F}_{2}$-linear map $K_{0}(\mathrm{C}) / 2 K_{0}(\mathrm{C}) \rightarrow{ }_{2} K_{1}(\mathrm{C})$. As $K_{0}(\mathrm{C})$ is the algebraic group completion of the monoid of isomorphism classes of objects in $\mathrm{C}$, all its elements have the shape $[X]-[Y]$ with $X, Y \in \mathrm{C}$ objects. In particular, for each $\beta_{i}$ pick

$$
\beta_{i}=\overline{[X]-[Y]}
$$

where $X, Y \in \mathrm{C}$ are objects. We have $q\left(\beta_{i}\right)=q([X])-q([Y])$ since $q$ is linear, and $q(x)=c(x, x)$, meaning that

$$
q([X])=s_{X, X}: \mathcal{D}(X) \otimes \mathcal{D}(X) \longrightarrow \mathcal{D}(X) \otimes \mathcal{D}(X),
$$

referring to the symmetry constraint of the virtual objects $V(\mathrm{C})$. In order to compute 
this map $s_{X, X}$, we can rely on

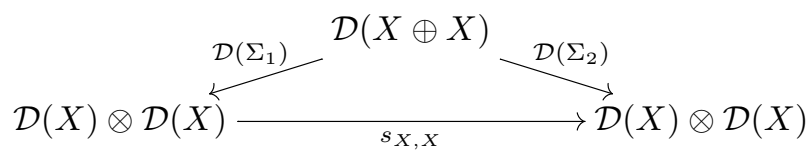

(which is Diagram A.3 in the special case of using the same object). The bottom arrow is the one we need, but the upper arrows come from $\mathcal{D}\left(\Sigma_{1}\right)$ resp. $\mathcal{D}\left(\Sigma_{2}\right)$, still in the notation loc. cit., which only differ by the swapping isomorphism in the middle term of $X \hookrightarrow X \oplus X \rightarrow X$, and the identity map on the two outer copies of $X$. In the attached homotopies, as in Equation A.5, also all vertices agree, and all outer edges agree, and only the three inner edges are different, because they differ exactly by the swapping of the two copies of $X$. For any object $X \in \mathrm{C}$, the swapping map

$$
X \oplus X \longrightarrow X \oplus X, \quad x_{1} \oplus x_{2} \mapsto x_{2} \oplus x_{1}
$$

is an automorphism, and thus functorially induces an automorphism of $\mathcal{D}(X \oplus X)$, meaning a homotopy, and this homotopy agrees with the one of $\mathcal{D}\left(\Sigma_{2}\right)^{-1} \circ \mathcal{D}\left(\Sigma_{1}\right)$. It follows that $s_{X, X} \in K_{1}(\mathrm{C})$ is just the automorphism attached to the swapping map of $X \oplus X$.

This finishes describing $\mathcal{T}\left(\pi_{0} V(\mathrm{C}), \pi_{1} V(\mathrm{C}),(0, c)\right)$ in detail. Next, we make the composition

$$
\operatorname{det}^{\text {strict }}: \mathrm{C}^{\times} \longrightarrow V(\mathrm{C}) \stackrel{\sim}{\longrightarrow} \mathcal{T}\left(\pi_{0} V(\mathrm{C}), \pi_{1} V(\mathrm{C}),(h, c)\right)
$$

a determinant functor. It is already a functor, and is taking values in a skeletal and strictly associative Picard groupoid. Explicitly:

Observation 1. The functor $\mathrm{det}^{\text {strict }}$ has the following properties:

1. on the level of objects, it sends $X \in \mathrm{C}$ to its $K_{0}$-class $[X] \in K_{0}(\mathrm{C})$,

2. on the level of automorphisms $\varphi: X \rightarrow X$, it sends $\varphi$ to its $K_{1}$-class $[X, \varphi] \in$ $K_{1}(\mathrm{C})$.

Here we use that $K_{0}(\mathrm{C})$ has the well-known explicit description of being the free abelian group on isomorphism classes of objects in $C$ (and quotient out $[X]=\left[X^{\prime}\right]+$ $\left[X^{\prime \prime}\right]$ whenever an exact sequence $X^{\prime} \hookrightarrow X \rightarrow X^{\prime \prime}$ exists); and that $K_{1}(\mathrm{C})$ contains canonical elements attached to automorphisms of objects (see for example [Wei13, Chapter IV, Exercise 8.7, or Example 9.6.2]). If $\mathrm{C}=P_{f}(R)$ is the category of finitely generated projective modules over a ring $R$, the group $K_{1}$ is actually generated by the classes coming from such automorphisms (this can also be used to give a presentation of the $K_{1}$-group; there is a discussion of this in [Nen98]).

We do not get a good description of morphisms on all of $\mathrm{C}^{\times}$, since isomorphisms between different objects will be sent to the class of the automorphism gotten once having pre- and post-composed the concrete choices of isomorphisms between the objects of $C$ and our choices tacitly made when picking a skeleton earlier, so this will not admit a choice-free description. Certainly, if one only needs to do a computation on finitely many objects, all this data can be chosen concretely and unravelled.

To promote det ${ }^{\text {strict }}: \mathrm{C}^{\times} \rightarrow \mathcal{T}\left(\pi_{0} V(\mathrm{C}), \pi_{1} V(\mathrm{C}),(0, c)\right)$ to a genuine determinant functor, it only remains to transport the datum $\mathcal{D}(\Sigma)$ attached to exact sequences $\Sigma$. 
However, given $\Sigma: G^{\prime} \hookrightarrow G \rightarrow G^{\prime \prime}$ in C, then under the equivalence of Equation A.6 in the isomorphism

$$
\mathcal{D}(\Sigma): \mathcal{D}(G) \stackrel{\sim}{\longrightarrow} \mathcal{D}\left(G^{\prime}\right) \underset{\mathrm{P}}{\otimes} \mathcal{D}\left(G^{\prime \prime}\right)
$$

both sides are the same because the Picard groupoid is skeletal. Thus, $\mathcal{D}(\Sigma)$ really only defines an automorphism of an object after going all to $\mathcal{T}\left(\pi_{0} V(\mathrm{C}), \pi_{1} V(\mathrm{C}),(0, c)\right)$. Such an automorphism is an element $B_{\Sigma} \in K_{1}(\mathrm{C})$. Equation A.2 now translates to the cocycle type identity

$$
B_{G_{2} \hookrightarrow G_{3} \rightarrow G_{3} / G_{2}}-B_{G_{1} \hookrightarrow G_{3} \rightarrow G_{3} / G_{1}}+B_{G_{1} \hookrightarrow G_{2} \rightarrow G_{2} / G_{1}}-B_{G_{2} / G_{1} \hookrightarrow G_{3} / G_{1} \rightarrow G_{3} / G_{2}}=0 .
$$

Observation 2. The functor $\operatorname{det}^{\text {strict }}$ is a universal determinant functor.

This holds because we constructed it from a universal one by symmetric monoidal equivalence (Definition A.2). Summarizing, this shows that at least abstractly there is a strict model for Deligne's universal determinant functor. Of course, in concrete terms, picking a genuine skeleton of $V(\mathrm{C})$ can be hard or impossible, depending on $\mathrm{C}$. For $\mathrm{C}=\operatorname{Vect}_{f}(k)$ being finite-dimensional vector spaces over a field, it can be done. Just sketching this, for each $n \in K_{0}(k)=\mathbb{Z}$ pick the object $X:=k^{n}$ if $n \geqslant 0$ and $k^{-n}$ for $n<1$, along with the standard basis. Then for an arbitrary finite-dimensional vector space picking a basis amounts to making the isomorphism to some $k^{n}$ explicit. The symmetry constraint can be computed using Equation A.7. One gets the standard Koszul type sign of the determinant line by observing that the matrix of the swapping map goes to +1 or -1 in $K_{1}$, depending on the rank (see [Wei13, Chapter III, Example 1.2.1]). Finally, working out the $B_{\Sigma} \in K_{1}(\mathrm{C})$ yields exactly the well-known rules for the top exterior power of the usual graded determinant line (restricted to this skeleton).

\section{References}

[Bau91] H. Baues, Combinatorial homotopy and 4-dimensional complexes, De Gruyter Exp. Math., vol. 2, Walter de Gruyter \& Co., Berlin, 1991, With a preface by Ronald Brown. MR 1096295

[BCC93] M. Bullejos, P. Carrasco, and A.M. Cegarra, Cohomology with coefficients in symmetric cat-groups. An extension of Eilenberg-Mac Lane's classification theorem, Math. Proc. Cambridge Philos. Soc. 114 (1993), no. 1, 163-189. MR 1219923

[BL04] J. Baez and A. Lauda, Higher-dimensional algebra. V. 2-groups, Theory Appl. Categ. 12 (2004), 423-491. MR 2068521

[Bre11] M. Breuning, Determinant functors on triangulated categories, J. KTheory 8 (2011), no. 2, 251-291. MR 2842932

[CC96] P. Carrasco and A.M. Cegarra, (Braided) tensor structures on homotopy groupoids and nerves of (braided) categorical groups, Comm. Algebra 24 (1996), no. 13, 3995-4058. MR 1414569

[CK07] A.M. Cegarra and E. Khmaladze, Homotopy classification of braided graded categorical groups, J. Pure Appl. Algebra 209 (2007), no. 2, 411-437. MR 2293318 
[Del87] P. Deligne, Le déterminant de la cohomologie, Current trends in arithmetical algebraic geometry (Arcata, Calif., 1985), Contemp. Math., vol. 67, Amer. Math. Soc., Providence, RI, 1987, pp. 93-177. MR 902592 (89b:32038)

[DGNO10] V. Drinfeld, S. Gelaki, D. Nikshych, and V. Ostrik, On braided fusion categories. I, Selecta Math. (N.S.) 16 (2010), no. 1, 1-119. MR 2609644

[Dur77] A. Durfee, Bilinear and quadratic forms on torsion modules, Adv. Math. 25 (1977), no. 2, 133-164. MR 480333

[EML53] S. Eilenberg and S. Mac Lane, On the groups $H(\Pi, n)$. I, Ann. of Math. (2) 58 (1953), 55-106. MR 0056295

[GJ16] C. Galindo and N. Jaramillo, Solutions of the hexagon equation for abelian anyons, Rev. Colombiana Mat. 50 (2016), no. 2, 273-294. MR 3605649

[GMdR02] A.R. Garzón, J.G. Miranda, and A. del Río, Tensor structures on homotopy groupoids of topological spaces, Internat. Math. J. 2 (2002), no. 5, 407-431. MR 1890928

[Isb69] J.R. Isbell, On coherent algebras and strict algebras, J. Algebra 13 (1969), 299-307. MR 0249484

[JO12] N. Johnson and A. Osorno, Modeling stable one-types, Theory Appl. Categ. 26 (2012), No. 20, 520-537. MR 2981952

[JS86] A. Joyal and R. Street, Braided monoidal categories, Macquarie Mathematical Reports (1986), no. 860081.

[JS93] _ Braided tensor categories, Adv. Math. 102 (1993), no. 1, 2078. MR 1250465

[Lap83] M. Laplaza, Coherence for categories with group structure: an alternative approach, J. Algebra 84 (1983), no. 2, 305-323. MR 723395

[ML52] S. Mac Lane, Cohomology theory of Abelian groups, Proceedings of the International Congress of Mathematicians, Cambridge, Mass., 1950, vol. 2, Amer. Math. Soc., Providence, R. I., 1952, pp. 8-14. MR 0045115

[Nen98] A. Nenashev, $K_{1}$ by generators and relations, J. Pure Appl. Algebra 131 (1998), no. 2, 195-212. MR 1637539

[NSW08] J. Neukirch, A. Schmidt, and K. Wingberg, Cohomology of number fields, second ed., Grundlehren Math. Wiss. [Fundamental Principles of Mathematical Sciences], vol. 323, Springer-Verlag, Berlin, 2008. MR 2392026

[Pat12] D. Patel, de Rham epsilon factors, Invent. Math. 190 (2012), no. 2, 299-355. MR 2981817

[Qui73] D. Quillen, Higher algebraic K-theory. I, Algebraic K-theory, I: Higher $K$-theories (Proc. Conf., Battelle Memorial Inst., Seattle, Wash., 1972), Springer, Berlin, 1973, pp. 85-147. Lecture Notes in Math., Vol. 341. MR 0338129 (49 \#2895)

[Qui99] F. Quinn, Group categories and their field theories, Proceedings of the Kirbyfest (Berkeley, CA, 1998), Geom. Topol. Monogr., vol. 2, Geom. Topol. Publ., Coventry, 1999, pp. 407-453. MR 1734419 
[Sín75] H.X. Sính, Gr-catégories (thesis, handwritten manuscript), Université Paris 7, https://pnp.mathematik. uni-stuttgart.de/lexmath/ kuenzer/sinh.html, 1975.

[Sri96] V. Srinivas, Algebraic K-theory, second ed., Progr. Math., vol. 90, Birkhäuser Boston, Inc., Boston, MA, 1996. MR 1382659

[Var] Various, Braided 2-Groups from Lattices, https://golem.ph.utexas . edu/category/2015/01/integral_octonions_part_12.html.

[Wal63] C.T.C. Wall, Quadratic forms on finite groups, and related topics, Topology 2 (1963), 281-298. MR 156890

[Wei94] C. Weibel, An introduction to homological algebra, Cambridge Stud. Adv. Math., vol. 38, Cambridge University Press, Cambridge, 1994. MR 1269324 (95f:18001)

[Wei13] _ The K-book, Grad. Stud. Math., vol. 145, American Mathematical Society, Providence, RI, 2013, An introduction to algebraic $K$-theory. MR 3076731

[Whi50] J.H.C. Whitehead, A certain exact sequence, Ann. of Math. (2) 52 (1950), 51-110. MR 35997

Oliver Braunling oliver.braeunling@math.uni-freiburg.de

Mathematical Institute, University of Freiburg, Ernst-Zermelo-Strasse 1, 79104 Freiburg im Breisgau, Germany 